\title{
Quaderni
}

QUADERNI Communication, technologies, pouvoir

76 | Automne 2011

Les promesses de la biodiversité

\section{Frédéric Forest, Freud et la Science : éléments} d'épistomologie

Economica/Anthropos, Paris, 2010

\section{Michel Daccache}

\section{(2) OpenEdition}

\section{Journals}

Édition électronique

URL : http://journals.openedition.org/quaderni/115

DOI : 10.4000/quaderni.115

ISSN : 2105-2956

Éditeur

Les éditions de la Maison des sciences de l'Homme

Édition imprimée

Date de publication : 1 septembre 2011

Pagination : 121-126

\section{Référence électronique}

Michel Daccache, «Frédéric Forest, Freud et la Science : éléments d'épistomologie », Quaderni [En ligne], 76 | Automne 2011, mis en ligne le 13 décembre 2012, consulté le 23 septembre 2020. URL : http:// journals.openedition.org/quaderni/115; DOI : https://doi.org/10.4000/quaderni.115 


\section{Livre}

\section{Freud et la Science: éléments d'épistomologie}

Frédéric Forest Economica/Anthropos Paris, 2010
Contrairement à ce que pourrait laisser penser le titre, l'ouvrage de Frédéric Forest, Freud et la science. Éléments d'épistémologie, ne pose pas directement la question de la scientificité de la psychanalyse (mesurée, par exemple, à l'aune de critères de validité à la Popper) mais celle des relations de Freud (et, eo ipso, de la psychanalyse) aux paradigmes scientifiques de son temps ${ }^{1}$. Ce faisant, il porte au jour la persistance de certains schèmes formels d'une discipline à l'autre tout en mettant l'accent sur la rupture majeure que constitue la psychanalyse sur le plan épistémologique par rapport à d'autres manières d'appréhender la vie psychique. Car, ce que tente l'auteur, c'est avant tout un « retour à Freud » visant à dégager une épistémologie psychanalytique qui, si elle n'a jamais été formulée en tant que telle, traverse en filigrane le corpus freudien. La figure du réseau en serait la trame secrète : loin d'être platement déterministe, la psychanalyse aurait en effet pour fondement une épistémologie complexe, non-linéaire et non-réductionniste, sensible à l'enchevêtrement et à la plurivocité des facteurs causaux.

Cette hypothèse, Frédéric Forest propose de la tester à travers un travail d'épistémologie historique visant à resituer la psychanalyse dans l'épistémè de son temps. Le XIX ${ }^{\mathrm{e}}$ siècle, rappellet-il, est marqué par un mouvement généralisé de « mise en visibilité », particulièrement perceptible en médecine comme en témoignent les progrès de la radiographie. Or, il ne faut pas l'oublier, avant d'être le père de la métapsychologie, Freud est neurologue ou, dirait-on aujourd'hui, anatomopathologiste. Et beaucoup des schèmes de pensée présents dans son œuvre, même la plus tardive, proviennent de ses premiers travaux réalisés par Michel Daccache Post-Doctorant, Cemagref Chercheur associé, CESSP 
dans le domaine de la biologie. Ainsi, en tant que neurologue, Freud participe à cette mise en visibilité généralisée, en élaborant par exemple une technique de coloration des tissus au chlorure d'or. Plus tard, à travers la psychanalyse, il y contribuera encore en mettant en jeu une série de couples oppositionnels (e.g. : contenu manifeste/ contenu latent; conscient/inconscient) visant à rendre compte des motifs cachés de l'action. Mais surtout, selon l'auteur, Freud importera de la neurologie une appréhension de la causalité en termes de réseaux, le modèle d'analyse proposé par Freud dans Esquisse d'une psychologie scientifique (1895) fonctionnant comme un « calque». Dans cet ouvrage, il s'agit en effet pour Freud de " réduire tous les processus psychiques à des états quantitativement déterminés de particules matérielles et de connexions de neurones » (p. 48). Freud y dépeint le cerveau comme un « système réticulaire » associant un atome notionnel (le neurone) et un flux (le flux électrique) à une fonction (de décharge), et donc justiciable d'une analyse « économique ». Or selon Frédéric Forest, la même structure se retrouve des années plus tard dans L'Interprétation du rêve, mais cette fois associée au triptyque représentations/affects/reconnaissance du désir. Par ailleurs, les hypothèses biologiques émises par Freud pour expliquer la vie et ses propositions au sujet du psychisme sont organisées autour des mêmes catégories binaires : " expansion-dégradation, liquide-solide, circulation-arrêt, couples d'opposés se rapportant à une opposition entre la vie et la mort [...]» (p. 31). Bref, entre les deux ouvrages, de la neurologie à la psychanalyse, et du cerveau à l'esprit, il y a transfert, transfert impliquant une sortie du déterminisme biologique. Car entre les deux modèles, il existe bien une homologie de structure, mais chacun se déploie à un niveau différent. Freud s'écarte ainsi des modèles explicatifs établissant des relations causales univoques et nécessaires entre caractéristiques physiques et psychiques, qu'il s'agisse des théories les plus triviales - la phrénologie ou la physiognomonie - au plus raffinées - théorie des zones hystérogènes de Jean-Martin Charcot ou lobotomie (cette dernière ayant valu un prix Nobel à Egas Moniz et Walter Rudolf Hess). L'opérateur qui garantit le passage d'un niveau à l'autre, ce sont probablement les travaux de Freud sur l'aphasie. Ce dernier trouble fonctionne en effet selon Frédéric Forest comme une "pierre de rosette théorique " ( $\mathrm{p}$. 101). En 1891, Freud rédige une Contribution à la conception des aphasies qui lui permet de " quitter définitivement le terrain biologique pour élaborer une théorie du langage fondée sur un appareil psychique véritable appareil à associer » (p.104). Ces travaux, s'ils ne relèvent pas encore de la psychanalyse, lèguent à celle-ci « un héritage graphique et une manière d'appréhender les phénomènes psychiques » (p. 101).

Cette approche réticulaire et complexe du psychisme qui serait selon Frédéric Forest le propre de la psychanalyse, c'est dans L'Interprétation $d u$ rêve qu'elle est le plus identifiable, cet ouvrage signant le basculement définitif vers la psychanalyse. En effet, « le rêve, principale formation de l'inconscient, est le terrain d'élection pour le développement de la théorie freudienne $d u$ psychisme et de l'interprétation en psychanalyse car tout y est affaire de liens. L'interprétation du rêve nous introduit également à toute une théorie de la circulation de l'affect et de sa régulation. Tous les éléments d'un système réticulaire sont pleinement développés et articulés. Ce système 
permet à Freud de rendre compte d'une causalité en réseau, c'est-à-dire multi et sur-déterminée » (p. 172). Le rêve agit donc comme un révélateur de la structure réticulée de l'inconscient. Un tel dévoilement n'est pas dépourvu d'effet sur la pratique analytique : afin d'interpréter le rêve (et cela vaut pour tout autre phénomène psychique) Freud invite à substituer un réseau à un autre, et plus exactement à remplacer « un premier réseau travaillé par la censure » par « le contenu latent où les véritables liens de causalité sont mis à jour» (p.173). Or, la relation contenu manifeste/ contenu latent est pluri-déterminée, une même représentation pouvant renvoyer à des sens multiples. C'est d'ailleurs le point de départ de l'associationnisme. Ainsi, avec l'interprétation du rêve et la pratique de l'association, on entre de plain-pied dans cette épistémologie freudienne annoncée par Frédéric Forest.

Cependant, remarque l'auteur, appréhender les phénomènes psychiques sur le mode du réseau ne garantit en rien contre le réductionnisme. Demeurent deux risques, dont Frédéric Forest décrit les manifestations historiques et contre lesquels il met en garde : la réification et la linéarité. Dans le premier cas, le danger est de rendre concret un élément abstrait ou de rendre corporel ce qui est psychique, qu'il s'agisse du réseau, de la fonction ou du fluide. L'auteur évoque ainsi Wilhelm Reich et son « orgone » ainsi que Pierre Janet et son «énergie psychique». Dans le second cas, il s'agit d'établir un lien nécessaire entre deux éléments d'ordre psychique. L'auteur pense ici à Otto Rank qui, à partir du seul « traumatisme de la naissance », prétend rendre compte d'à peu près tous les troubles psychiques. Mais, rappelle Frédéric Forest, Freud a lui-même pu à l'occasion céder à cette tentation, en établissant par exemple dans Sur le rêve, des liens nécessaires entre certains symboles et certaines significations.

Une fois mises au jour cette épistémologie freudienne et sa genèse, l'auteur s'aventure sur le terrain des pratiques thérapeutiques associées aux différentes manières d'appréhender le psychisme. S'il se pose en défenseur de la cure par la parole, il met à nouveau en garde contre le péché de réductionnisme, évoquant par exemple l'utopie thérapeutique d'une « communication-lubrification » dans laquelle « la métaphore de la communication généralisée peut se réifier dans une pure équation où communication égale guérison » (p. 204). Il pense notamment à la Network Therapy, à mille lieues de laquelle se situerait la psychanalyse, car " si l'austère analyse freudienne repose sur un postulat semblable - une circulation des affects et une communication des représentations - ses conditions (le setting) instaurent cependant un mode opératoire particulier centré autour de la reconnaissance de l'inconscient et fondé sur l'idée d'un déterminisme en réseau » (p.205). C'est donc bien son attention à l'enchevêtrement des représentations qui singularise la psychanalyse et l'élève, selon Frédéric Forest, au rang de discipline de la complexité s'opposant à la fois aux neurosciences et aux thérapies comportementales.

$\mathrm{Au}$ final, ce que propose Frédéric Forest est un travail d'épistémologie historique original, soutenu par une fine connaissance du corpus freudien et une familiarité manifeste avec la pratique psychanalytique. On reconnaîtra dans la démarche générale adoptée par l'auteur certains traits de la méthode historienne associée au nom 
de M. Foucault, notamment dans la volonté de reconstruire une épistémè, ou plutôt de rendre compte du basculement d'un régime épistémique à un autre. Au-delà des discours savants, Frédéric Forest est attentif à un contexte culturel plus large, ne négligeant par exemple en rien le rôle des imaginaires techniques - eau, électricité, téléphonequi constituent un véritable trésor de métaphores pour mieux se figurer la vie de l'esprit.

L'ouvrage de Frédéric Forest présente une autre originalité : sa forte dimension graphique. Richement imagées, les représentations visuelles ont tour à tour le rôle de preuves et d'illustrations. Ainsi, la présentation des différents schémas réalisés de la main même de Freud donne une consistance certaine à l'idée selon laquelle le modèle élaboré dans l'Esquisse aurait fonctionné comme un calque. Preuve également qu'il existerait bien une raison graphique à l'œuvre dans l'ensemble du corpus freudien. Dans un autre registre, le basculement d'un mode de penser à l'autre est illustré par l'auteur à travers Une leçon clinique à la Salpêtrière (1887), toile d'André Brouillet dépeignant la présentation par Charcot d'un cas de grande hystérie. Les deux paradigmes sont en effet en présence : au centre de la toile on distingue les instruments d'examen, à savoir un marteau-réflexe (qui matérialise la causalité linéaire simple type stimulus-réponse) et une bobine électrique (symbole de l'approche réticulaire).

On pourrait cependant reprocher à l'auteur de trop se cantonner à une histoire des idées pure, désincarnée et éthérée. Bien que son propos ne soit pas de réaliser une sociologie ou une histoire de la psychanalyse, une attention plus grande aux conditions concrètes de production des textes, aurait permis d'échapper à l'exégèse à laquelle il semble parfois céder. Un tel travail conduirait peut-être également à relativiser quelque peu la rupture épistémologique identifiée dans le champ scientifique. En effet, ce basculement du simple au complexe pourrait bien n'être que le produit d'une illusion historiographique, les modalités de causalité linéaire étant plus simples à repérer dans les archives, alors que certaines approches complexes de la santé mentale - tel l'hygiénisme - semble avoir existé bien avant la naissance de la psychanalyse.

On pourrait d'ailleurs se demander si ce que l'auteur désigne comme une épistémologie complexe, ne se réduit pas en vérité à une épistémologie linéaire « compliquée », raffinée par la prise en compte des histoires personnelles et donc de la grande diversité des trajectoires empruntées par les affects. On a de plus le sentiment que chez Freud, les chaînes de causalité restent, malgré tout univoques, dirigées dans un certain sens et donc orientées dans le temps (alors que dans une épistémologie véritablement complexe, la notion de causalité tend à être remplacée par celle de corrélation, ce qui n'est pas dépourvu d'effets sur la manière d'appréhender le temps). Enfin, on peut s'interroger : en autonomisant le domaine de l'esprit, Freud ne le coupe-t-il pas radicalement du biologique ? Jean Piaget par exemple, chez qui développements psychique et biologique sont profondément associés, serait alors un meilleur représentant d'une épistémologie complexe. Quoi qu'il en soit, on peine parfois à dissocier l'épistémologie freudienne d'une simple complication des causalités. Certains arguments méritent à ce sujet d'être développés. La notion d'après-coup, 
dont il est rappelé qu'elle relève d'une causalité circulaire, constitue un élément important dans une telle démonstration. De même, on sait que chez Freud, il règne dans l'inconscient un temps différent, voire pas de temps du tout, ce qui pourrait là aussi donner lieu à une argumentation intéressante. Enfin, le concept de pulsion, qui reste l'unité de base de la psychanalyse, et qui est largement abordé dans le livre, appartient également aux règnes du psychique et du biologique. Autant d'exemples qui abondent dans le sens d'une épistémologie complexe.

Cette mise au point épistémologique reste en tout cas bienvenue. Freud et la psychanalyse en effet, sont aujourd'hui pris en tenaille entre deux types de discours, destructeurs ou hagiographiques. Or, ces deux types de discours ont en commun un rapport problématique à l'objet « Freud», un manque d'intérêt pour les questionnements de nature épistémologique, et une méconnaissance manifeste de la pratique psychanalytique comme de la clinique. S'il existe bien une actualité scientifique dans le domaine ${ }^{2}$, ce sont surtout les brûlots et les controverses qu'ils font naître qui occupent le devant de la scène et mobilisent les énergies ${ }^{3}$. Avec son ouvrage, Frédéric Forest a le mérite de faire d'une pierre deux coups : contribuer de manière informée et inventive à l'effort de réflexivité de la psychanalyse tout en proposant un texte critique et engagé, aux implications politiques profondes. En effet, l'opposition linéaire/réticulaire qui structure le texte, au-delà de sa portée conceptuelle, pose la question des modes de « traitement » du psychisme. Alors que l'approche réductionniste conduit inévitablement à des interventions mécaniques (via une instrumentation matérielle ou des produits chimiques), l'approche réticulaire, elle, plaide en faveur d'une cure par la parole sensible à la singularité de chaque histoire. 


\section{$\mathrm{N} \cdot \mathrm{O} \cdot \mathrm{T} \cdot \mathrm{E} \cdot \mathrm{S}$}

1. Au sujet de la scientificité de la psychanalyse, on pourra se reporter à l'ouvrage suivant : Vannina Micheli-Rechtman, La psychanalyse face à ses détracteurs, Paris, Aubier, 2007, 289 p.

2. Cf. la publication récente de textes inédits de Norbert Elias sur le sujet (Au-delà de Freud. Sociologie, psychologie, psychanalyse, Paris, La Découverte, 2010, 216 p.) et de la thèse de Samuel Lézé (L'autorité des psychanalystes, Paris, PUF, 2010, 216 p.).

3. On pense évidemment à la vive polémique suscitée par la publication de deux textes de Michel Onfray (Le crépuscule d'une idole, Paris, Grasset, 2010, 624 p. et Apostille au crépuscule. Pour une psychanalyse non-freudienne, Paris, Grasset, 2010, 224 p.). Frédéric Forest a d'ailleurs participé au débat en publiant avec François Pommier un article dans Le Monde (Onfray, le chapitre manquant, 01/06/10, disponible à l'URL suivante : http://www.lemonde.fr/idees/ article/2010/06/01/onfray-le-chapitre-manquant-parfrederic-forest-et-francois-pommier_1365736_3232. html). 